\title{
Isolation of Nanocrystalline Cellulose from oil palm empty fruit bunch - A response surface methodology study
}

\author{
Yee Kai Song ${ }^{1,2}$, Irene Mei Leng Chew ${ }^{3}$, Thomas Shean Yaw Choong ${ }^{1}$, Jully $\operatorname{Tan}^{2}$ and Khang Wei $\operatorname{Tan}^{2}$ \\ ${ }^{1}$ Faculty of Engineering, University Putra Malaysia, 43400 UPM, Serdang, Selangor Darul Ehsan, Malaysia \\ ${ }^{2}$ Faculty of Engineering, Technology and Built Environment, UCSI University, Cheras, 56000 Kuala Lumpur, Malaysia. \\ ${ }^{3}$ School of Engineering, Monash University, 46150 Bandar Sunway, Selangor Darul Ehsan, Malaysia.
}

\begin{abstract}
The research work studied the extraction of NanoCrystalline Cellulose (NCC) from oil palm empty fruit bunch (EFB), with aid of Response Surface Methodology (RSM). Particle size analysis using Malvern Zetasizer had confirmed the extracted NCC fall within the desired nano scaled range. The impact of three input parameters, namely concentration of $\mathrm{NaOH}$ solution during alkaline treatment, concentration of $\mathrm{H}_{2} \mathrm{SO}_{4}$ solution during acid hydrolysis, and duration for acid hydrolysis on NCC particle were investigated. From ANOVA study, it had suggested that the current RSM model is significant to interpret the interaction among the all three input parameters.
\end{abstract}

\section{Introduction}

Back to 1951, Rånby (1951) demonstrated a fundamental piece of work which stated that native cellulose fibers can be degraded using sulphuric acid as hydrolysis agent [1]. In 50 years apart into $21^{\text {st }}$ century, Rånby’s work in acid hydrolysis on native cellulose had been a solid guidance on blossoming numerous cellulose particle extraction from biomass sources, to name a few, tunicate [2], ramie [3], and wood [4].

Empty fruit bunch (EFB) is a by-product from mills to obtain valuable oil. In most tropical countries, especially Malaysia, EFB from palm oil tree is one of the abundantly available fibrous biomass materials, which is always regarded as waste and extremely low in commercial value. Some general applications on EFB are pulp production, used as stabilizing agent for soil, horticulture, or used as fu.el for power generation [5]. Applications can be critically many; however the commercial viability is trivial. Perhaps, by hydrolyzing EFB into fermented saccharides, which can be further developed into valuable ethanol [6] seems to be a good idea to increase ultilization value of EFB. Though, the high handling cost, large inventory spaces needed due to its bulkiness, purity of ethanol produced, and dependency of ethanol price to the market could jeopardize its commercial viability. These factors are leading to high demand of cost effectiveness in NCC extraction from any form of biomass in order to have high commercial viability in the future.

As aforementioned, sulphuric acid hydrolysis is the most fundamental chemical method to isolate crystalline region in any form of cellulosic material [1]. With such specific method to produce NanoCrystalline Cellulose (NCC) from cellulose, it imposed a setback to the practicality of NCC on industry that required high processing temperature, which is caused by reduced thermal stability, or thermal degradation prior to acid hydrolysis. Poletto et al. [7] reported that lesser the percentage of crystalline extracted from wood with association with smaller crystalline size could lead to accelerated thermal degradation. This finding is essential to justify the necessity of searching an alternative method to extract NCC with lower particle size. The study on the role of acid particularly on its impact on the isolation of crystalline particle is crucial, because from the magnitude of degradation it basically determine the size of NCC extracted. Response surface methodology (RSM) was commonly used as an optimization tool due to its well establishment in examining experiment variables, deriving model, and factors used in the optimization parameters back in 1983 [8]. In the current study, a foundation was laid down for further research by studying the significance and interactions among the three input parameters, i.e. concentration of $\mathrm{NaOH}$ solution during alkaline treatment, concentration of $\mathrm{H}_{2} \mathrm{SO}_{4}$ solution during acid hydrolysis, and duration for acid hydrolysis, on their impact to determine the NCC particle size. It provided an insight of how to reduce the involvement of acid during NCC isolation to obtain finer NCC particles.

\section{Methodology}

\subsection{Isolation of NCC}




\subsubsection{Preparation of empty fruit bunches}

Empty fruit bunches (EFB) were grinded to a size of approximately $10 \mathrm{~mm}$. The grinded EFB were washed for 10 min to remove dust, dirt, wax, and impurities on the surface of EFB. $10 \mathrm{~g}$ of grinded EFB were dispersed in $800 \mathrm{~mL}$ distilled water on a stirring hot plate at a constant temperature of $50^{\circ} \mathrm{C}$ for $2 \mathrm{~h}$ to strengthen the outcome of this pretreatment. The suspension was then sieved through a $300 \mu \mathrm{m}$ mesh size sieve. Followed by that, the sieved EFB was treated with $800 \mathrm{~mL}$ of $10-30 \% \mathrm{NaOH}$ solution at a temperature of $80^{\circ} \mathrm{C}$ for $6 \mathrm{~h}$, and then the EFB was washed with distilled water until $\mathrm{pH} 7$ was obtained. This process is generally known as the alkaline treatment to remove hemicelluloses along with partial lignin on the EFB. The alkaline treated EFB was dried in the oven for $24 \mathrm{~h}$ at a temperature of $70{ }^{\circ} \mathrm{C}$ to remove moisture content. Next, bleaching process was done to remove the lignin, i.e. $3 \mathrm{~g}$ of the alkaline-treated EFB was dispersed in $350 \mathrm{~mL}$ of distilled water that is mixed with $10 \mathrm{~mL}$ of hydrogen peroxide $\left(\mathrm{H}_{2} \mathrm{O}_{2}\right)$ and $20-22$ drops of glacial acetic acid $\left(\mathrm{CH}_{3} \mathrm{COOH}\right)$. The mixture was stirred at medium temperature of $70{ }^{\circ} \mathrm{C}$ for $7 \mathrm{~h}$, followed up by sieving in a $60 \mu \mathrm{m}$ mesh size sieve. Then, the mixture was washed with distilled water until $\mathrm{pH} 7$ was obtained, and dried in oven at $70^{\circ} \mathrm{C}$ for $24 \mathrm{~h}$ for further process.

\subsubsection{Extraction of NCC}

Sulphuric acid hydrolysis was conducted to extract NCC from the treated EFB. $0.3 \mathrm{~g}$ of bleached EFB was dispersed in $4 \mathrm{~mL}$ consist of $56-64 \%$ weight of sulphuric acid $\left(\mathrm{H}_{2} \mathrm{SO}_{4}\right)$ solution at a constant temperature of $45^{\circ} \mathrm{C}$ for $1-3 \mathrm{~h}$. The hydrolysed samples were washed using distilled water until $\mathrm{pH} 5$ is obtained. After washing, centrifugal was used to separate the NCC from the heavy component at $3000 \mathrm{rpm}$ for $10 \mathrm{~min}$.

\subsection{Characterization and Analysis}

\subsubsection{Particle Size Analysis}

The extracted NCC was dispersed in distilled water and analyzed using Malvern Zetasizer, Nano ZS series. The measurement was repeated twice and z-average was generally regarded as particle average size in this study.

\subsubsection{Response Surface Methodology (RSM)}

Response surface methodology (RSM) is statistical-based software which is useful to optimize the isolation process of NCC in this study. From preliminary experiment, three (3) independent variables which are concentration of alkaline used $(\mathrm{NaOH})$, concentration of acid used $\left(\mathrm{H}_{2} \mathrm{SO}_{4}\right)$, and duration of sulphuric acid hydrolysis were being investigated. Box-Behnken Design (BBD) was employed to reduce the complexity which only requires few significant points from three level factorial arrangements. Hence, the total number of experiments needed is 17 , and the z-average is the only response in this study. The boundary limit for the three input variables are $10-30 \%, 56-64 \%$ and $1 \mathrm{~h}$ to $3 \mathrm{~h}$, for concentration of alkaline used $(\mathrm{NaOH})$, concentration of acid used $\left(\mathrm{H}_{2} \mathrm{SO}_{4}\right)$, and duration of sulphuric acid hydrolysis, respectively. The resulted response surface model is validated statistically with analysis of variance, or in abbreviation ANOVA.

\section{Result and Discussion}

\subsection{Particle Size Analysis}

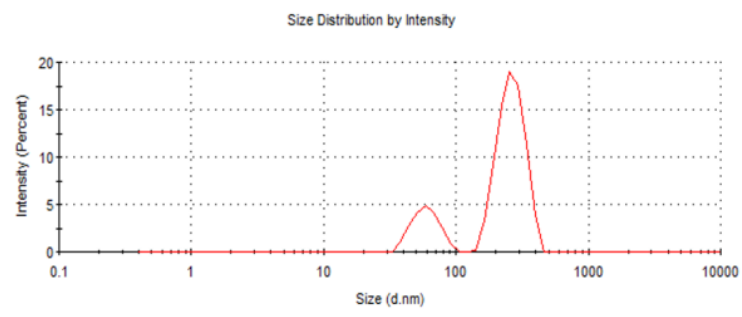

Figure 1. Particle Size Analysis by Malvern Zetasizer, Nano ZS Series.

For the first run of experiment, NCC was obtained with the following condition i.e. $1 \mathrm{M} \mathrm{NaOH}$ solution, $60 \%$ $\mathrm{H}_{2} \mathrm{SO}_{4}$ solution and $2 \mathrm{~h}$ hydrolysis time. As shown in Fig. 1, the analysis indicated that the size distribution by intensity has two peaks, recorded as $59.79 \mathrm{~nm}$ for peak 1 , and $264.9 \mathrm{~nm}$ for peak 2. As a whole, the z-average was recorded as $420.6 \mathrm{~nm}$. It was reported that NCC is a typical elongated rod-like shape with diameter ranging from $10 \mathrm{~nm}$ to $100 \mathrm{~nm}$ while length ranging from $100 \mathrm{~nm}$ to $1000 \mathrm{~nm}$ [9]. Polydispersity index (PDI) value of 0.528 was obtained due to non-uniformity of the particle size. It was theorized as in the effect of the orientation of NCC across the dynamic light scattering during the measurement. According to Stoke-Einstein theory [10], the larger particle diffuses in a slower rate compared to the smaller particle. For instance, when NCC diffuses across the aqueous medium in horizontal orientation against the light source, the size interpretation by Zetasizer is smaller, due to a faster speed detected for its difussion. Similarly, if NCC diffuses across the aqueous medium in vertical orientation against the light source, the size interpretation by Zetasizer will be bigger. It is theorized that the larger surface area contribute to higher resistances in the direction of diffusion, resulting in lower speed of NCC diffusion. This phenomenon is illustrated in Fig. 2. In recognition of this assumption, particle size measurement was repeated for samples in RSM study.
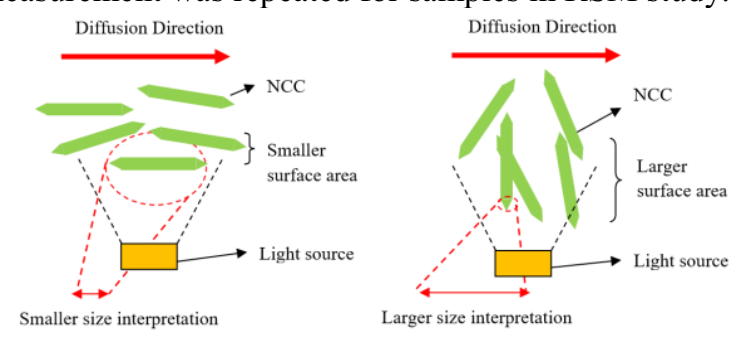

Figure 2. Effect of surface area in the direction of diffusion leading to size interpretation by Zetasizer. 


\subsection{Response Surface Methodology}

With exactly defined domain of independent experimentation variables, a much simpler illustration (Fig. 3) can be done to understand vividly on how these variables could be fitted into Box-Behnken Design (BBD) model which required only 17 sets of experiment data for process optimization. The box is the boundary limit controlled at only 3 level figures as in $-1,0$, and +1 to the experimental domain that represent the minimum and maximum value obtained from the 17 sets of experiment data. Three-dimensional (3D) contour plot were generated by RSM. In general, the 3D graph determined the overall contribution of the input variables to the $\mathrm{z}$ average. Green region signifies that input variables had less impact to the output variables. The significance of input variables increases with intensification of colorization from green region to dark blue region progressively. The stationary point, which is denoted by dark blue region on lower part of wedge-like 3D graph is the optimized point. Hence, it can be justified that both concentration of $\mathrm{NaOH}$ and $\mathrm{H}_{2} \mathrm{SO}_{4}$ are critical parameters in altering z-average. At low concentration of $\mathrm{NaOH}$ and low concentration of $\mathrm{H}_{2} \mathrm{SO}_{4}$, the $\mathrm{z}$-average diameter is of the highest point throughout the $3 \mathrm{D}$ contour plot. This result is valid as both of $\mathrm{NaOH}$ and $\mathrm{H}_{2} \mathrm{SO}_{4}$ had to perform their function in isolating of NCC. Without utilization of $\mathrm{NaOH}$, the removal of hemicelluloses layer and partial lignin would had become a bottleneck process to proceed further into inner layer of EFB. Without sufficient of $\mathrm{H}_{2} \mathrm{SO}_{4}$, the extraction of NCC will be affected and hence resulted in larger particle size.

Further steps are taken to analyze the effect of duration of sulphuric acid hydrolysis on the z-average. Fig. 4 showed pattern changes as the duration of acid hydrolysis increases. Starting from the left to the right, the high-significant dark blue region expanded in
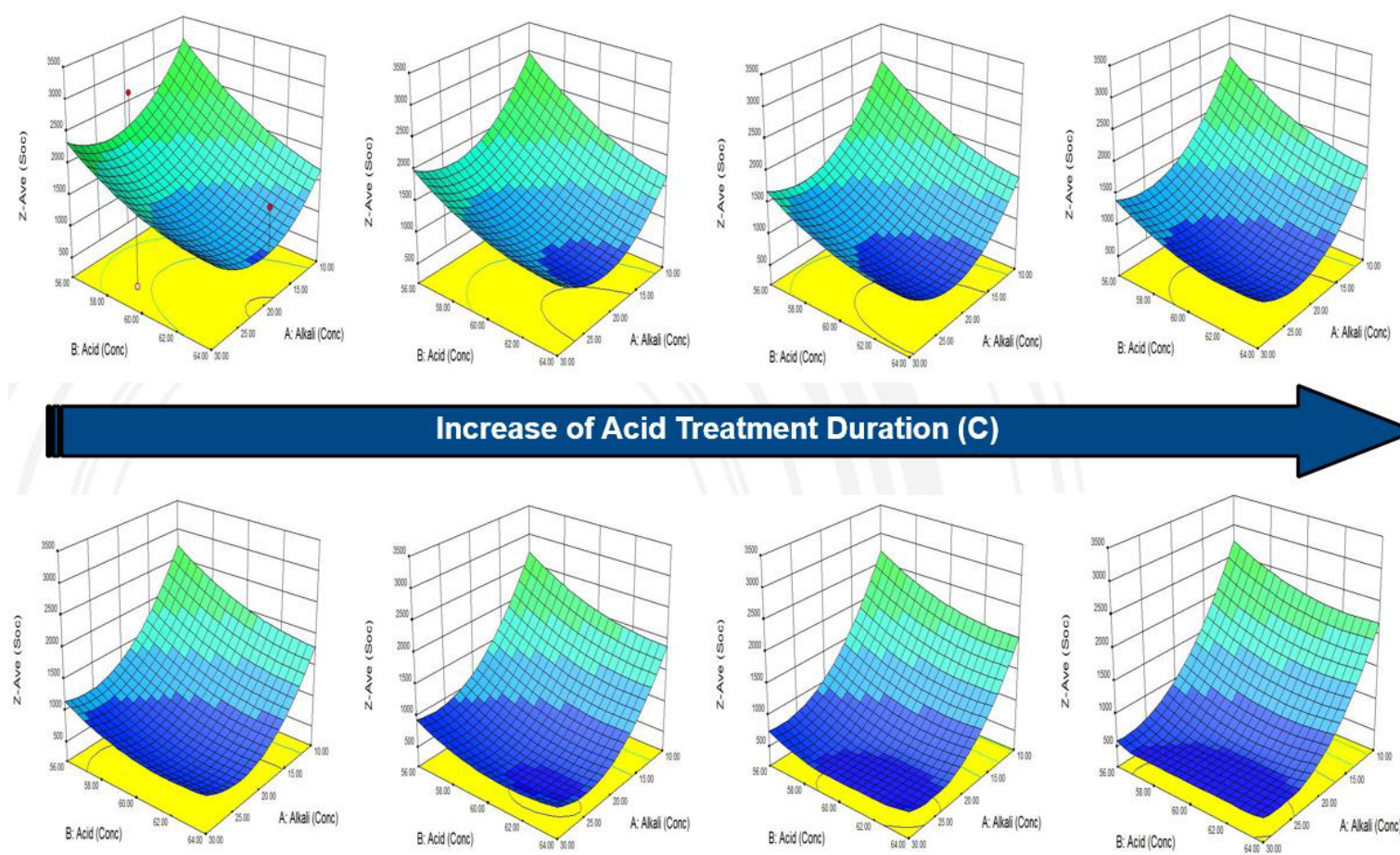

elongation form across $3 \mathrm{D}$ contour plot as the duration of acid hydrolysis increases. It also signifies that duration of acid hydrolysis is able to consolidate larger range of alkaline concentration and acid concentration in order to produce lower z-average diameter.

Table 1 tabulated data generated by RSM, which is known as ANOVA. It measure the significance and correlation between each studied input parameters. Pvalue is a numerical reference to validate the interactions among the three input variables. Statistically, it is desirable to have a P-value below 0.05 to justify a significant interactions. Following this "golden rule", interaction between $\mathrm{B}$ and $\mathrm{C}$, which are acid concentration and acid hydrolysis duration, owning a $\mathrm{p}$ value of 0.0042 that is much smaller than 0.05 . This has statistically-proven that the interaction between these two variables is the most critical parameters to determine the z-average.

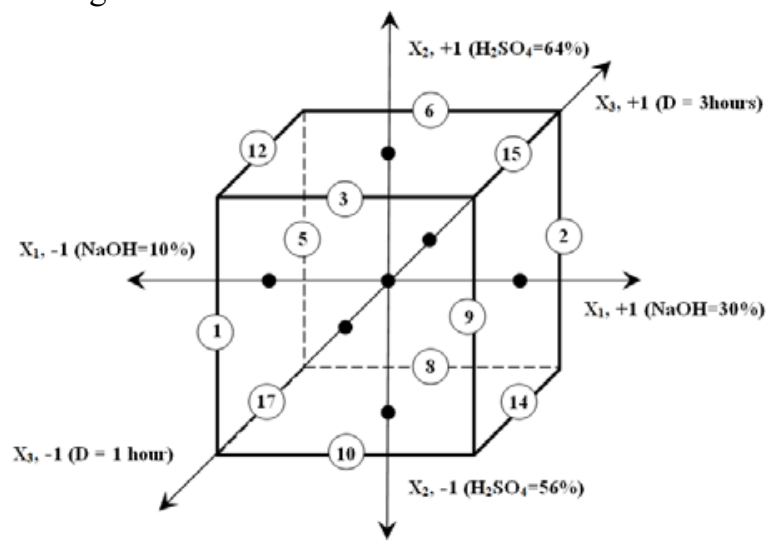

Figure 3. Illustration of three independent variables (X1: $\mathrm{NaOH}$ concentration, X2: H2SO4 concentration, X3: Duration of $\mathrm{H} 2 \mathrm{SO} 4$ hydrolysis) fitted in Box-Behnken Design (BBD).

Figure 4. Effect of duration of $\mathrm{H}_{2} \mathrm{SO}_{4}$ hydrolysis on the z-average progressively. 
Table 1. Analysis of Variance (ANOVA)

\begin{tabular}{lcccc}
\hline & \multicolumn{4}{c}{ Particle Size (nm) } \\
\cline { 2 - 5 } & $\begin{array}{c}\text { Sum of } \\
\text { Squares }\end{array}$ & $\begin{array}{c}\text { Deg. of } \\
\text { Freedom }\end{array}$ & F-value & P-value \\
\hline Model & $2.45 \times 10^{5}$ & 9 & 8.71 & 0.0141 \\
$\quad$ Lack of fit & 14817.13 & 3 & 12.35 & 0.0759 \\
$\quad$ Pure Error & 800.06 & 2 & - NIL - & - NIL - \\
Residual & 15617.19 & 5 & - NIL - & - NIL - \\
$\quad$ Total & $2.606 \times 10^{5}$ & 14 & - NIL - & - NIL - \\
Linear & & & & \\
A - Alkali Conc. & 534.65 & 1 & 0.17 & 0.6962 \\
B - Acid Conc. & 2949.12 & 1 & 0.94 & 0.3758 \\
C - Duration & 7638.48 & 1 & 2.45 & 0.1786 \\
Interaction & & & & \\
AB & 1879.22 & 1 & 0.6 & 0.473 \\
AC & 3186.6 & 1 & 1.02 & 0.3588 \\
BC & 77423.06 & 1 & 24.79 & 0.0042 \\
Quadric & & & & \\
A $^{2}$ & $1.105 \times 10^{5}$ & 1 & 35.39 & 0.0019 \\
B $^{2}$ & 25983.19 & 1 & 8.32 & 0.0344 \\
$\mathrm{C}^{2}$ & 3981.42 & 1 & 1.27 & 0.3101 \\
\hline
\end{tabular}

It was observed that the quadric relation of $\mathrm{A}^{2}$ resulted in value of 0.0019 , which is lower than 0.05 . When alkaline concentration is decreased, $\mathrm{z}$-average diameter is increased. It is due to the higher alkaline concentration, the higher the ability to dissolve hemicelluloses/lignin to a greater extent, hence exposing more cellulose crystalline region for acid hydrolysis.

Fig. 5 illustrated that the parity plot to display the closeness of actual value to the predicted value. The less deviation from the straight line indicates less deviation from the predicted value. It is a satisfactory correlation between experimental data and predictive data.

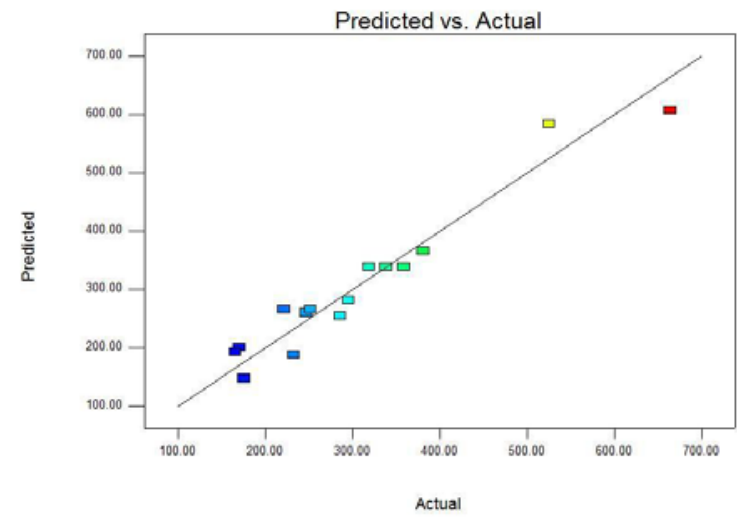

Figure 5. Parity plot displaying the distribution of experimental data against predicted data of the resulted y value.

However, it can observe that the increase duration of sulphuric acid hydrolysis would impose a major setback to be used as a deciding parameter in process optimization. Wang et al. [11] reported that at $64 \% \mathrm{wt}$ of $\mathrm{H}_{2} \mathrm{SO}_{4}$, insignificant amount of cellulose remained. This is attributed to complete hydrolysis of cellulose to soluble sugars and various by-products. The finding proved that excessive treatment using $\mathrm{H}_{2} \mathrm{SO}_{4}$ either in higher concentration or prolonged treatment duration, $\mathrm{NCC}$ yield percentage would not improved but it will be degraded into other products.
From the past decades, there are a lot of studies had been conducted in attempt to optimize the yield of NCC. Rånby [1] reported as high as $40 \%$ NCC yield at $22 \%$ wt of $\mathrm{H}_{2} \mathrm{SO}_{4}$ of prolonged treatment time. Not long later a study shown that $65 \%$ wt of $\mathrm{H}_{2} \mathrm{SO}_{4}$ are capable of extracting NCC but it is only limited to maximum of $30 \%$ yield [12]. Most recent advancement is that Bondeson and Oksman (2006) attempted to extract NCC straight from microcrystalline cellulose (MCC) via acid hydrolysis [13]. However, this approach was reported to have a wide range of yield percentage, from 0 to $95 \%$. Apart from unstable production of NCC, agglomeration phenomenon is also reported for above $47 \%$ yield of NCC. To authors' knowledge, development of NCC production via acid hydrolysis did not take off a significant leap forward since more than 6 decades ago. Hence, for sulphuric acid hydrolysis, this range is proposed to be the optimized condition for NCC extraction from EFB.

\section{Conclusion}

NCC was successfully isolated from EFB using a conventional sulphuric acid hydrolysis method. Zetasizer on particle size analysis using Zetasizer is a quick and feasible option which suggested the isolated NCC has particle size below $300 \mathrm{~nm}$. ANOVA suggested the conventional chemical approach, especially for $\mathrm{NaOH}$ concentration, $\mathrm{H}_{2} \mathrm{SO}_{4}$ concentration, and duration of $\mathrm{H}_{2} \mathrm{SO}_{4}$ hydrolysis, played a crucial part to determine the final particle size. This is significant as it provides a fundamental for future research to reduce involvement of acid, as well as other chemical involved.

\section{Acknowledgement}

The authors are thankful to Ministry of Higher Education (MOHE) for the financial support through ERGS (ERGS/1/2013/TK07/UCSI/03/1), and CERVIE for RGS (PROJ-IN-FETBE-015). The authors also thankful to the Institution of Engineers Singapore (IES) for the funding to develop part of the prototype.

\section{References}

1. Rånby, B. G., (1951). The Colloidal Properties of Cellulose Micelles.Discussions of the Faraday Society. 11, pp. $158-164$.

2. Kimura, F., Kimura, T., Tamura, M., Hirai, A., Ikuno, M. and Horii, F. (2005).Magnetic Alignment of the Chiral Nematic Phase of a Cellulose Microfibril Suspension. Langmuir. 21, pp. 2034 - 2037.

3. De Menezes, A. Jr., Siqueira, G., Curvelo,A. A. S., and Dufresne,A., (2009). Extrusion and Characterisation of Functionalised Cellulose Whiskers Reinforced Polyethylene Nanocomposites. Polymer. 50, pp. 4552 - 4563.

4. Beck-Candanedo, S., Roman, M. and Gray, D. G. (2005). Effect of Reaction Conditions on the Properties and Behaviour of Wood Cellulose 
Nanocrystal Suspensions.Biomacromolecules.6,pp 1048-1054.

5. Diego Piarpuzan, Julian A. Quintero, Carlos A. Cardona (2011) Empty fruit bunches from oil palm as a potential raw material for fuel ethanol production, Biomass and Bioenergy 35, 1130-1137.

6. Ohara, S., Kato, T., Fukushima, Y.and Sakoda, A., (2013). Selective Ethanol Production from Reducing Sugars in a Saccharide Mixture.J BiosciBioeng. 115(5), pp. 540 - 543.

7. Poletto, M., Zattera, A. J., Forte, M. M. C., and Santana, R. M. C. (2012). Thermal Decomposition of Wood: Influence of Wood Components and Cellulose Crystallite Size. Bioresource Technology. 109, pp. $148-153$.

8. Cheynier, V., Feinberg, M., Chararas, C and Ducauze, C. (1983). Application of Response Surface Methodology to Evaluation of Bioconversion Experimental Conditions. Appl. Env. Microbiol. 45, pp. 634-639.

9. Ruiz, M. M., Cavaillé, J. Y., Dufresne, A., Gérard, J. F., and Graillat, C. (2000).Processing and Characterization of New Thermoset Nanocomposites Based On Cellulose Whiskers. Compos Interfaces. 7(2), pp. 117-131.

10. Sharma, M., and Yashonath, S. (2007). Size Dependence of Solute Diffusity and Stoke-Einstein Relationship: Effect of Van Der Waals Interactions. Diffusion Fundamentals, pp. 11.1-11.5.

11. Wang, Q. Q., Zhu, J. Y. and Reiner, R. S. (2012). Approaching Zero Cellulose Loss in Cellulose Nanocrystal (CNC) production: Recovery and Characterization of Cellulosic Solid Residues (CSR) and CNC. Cellulose. 19, pp. 2033-2047.

12. Mukherjee, S. M. and Woods, H. J. (1953). X-ray and Electron Microscope Studies of the Degradation of Cellulose by Sulphuric Acid. Biochim Biophys Acta. 10, pp. 499-511.

13. Bondeson, D. A. M. and Oksman, K. (2006). Optimization of the Isolation of Nanocrystals From Microcrystalline Cellulose by Acid Hydrolysis. Cellulose. 13, pp. 171-180. 